\title{
BULGARIAN PHYSICIANS AND NURSES VS. ELECTRONIC HEALTH RECORDS AS A MAJOR ISSUE OF E-HEALTH
}

\author{
Iskra Mircheva $^{1}$, Martin Mirchev ${ }^{1}$, Veselinka Nestorova ${ }^{2}$ \\ ${ }^{1}$ Department of Social Medicine and Healthcare Organization, Faculty of Public Health, \\ Medical University of Varna \\ ${ }^{2}$ Department of Physiotherapy, Rehabilitation, Thalassotherapy, Occupational Diseases, \\ Faculty of Public Health, Medical University of Varna
}

\section{ABSTRACT}

INTRODUCTION: Despite the numerous e-health applications, e-health cannot be implemented without the development and implementation of electronic health records (EHRs). The main barriers to the deployment of its systems are the lack of funding, infrastructure, capacity and legal framework, as well as the insufficient computer literacy of medical staff.

AIM: The aim of this article is to assess the computer literacy of Bulgarian physicians and nurses, their attitude to e-health, especially EHRs as a major issue of e-health, including the ownership of medical data.

MATERIAL AND METHODS: An enquiry comprising 33 questions is used. Respondents of the study are physicians and nurses from five regional and university hospitals in the North-eastern region of Bulgaria. Some of the results are compared to those of a study, carried out in 1999 among the physicians in Bulgaria. Several signs are discussed: self-evaluation of the experience in using computers, general attitude to eHealth, attitude to EHRs, including ownership of medical data.

RESULTS: The overall self-evaluation in using computers is 4.16 for physicians and 3.74 for nurses $(\max =6.00)$. The greatest need for additional training is in the use of statistical $(47.98 \%$ of the physicians and $39.24 \%$ of the nurses) and specialized software (48.34\% of the physicians and $49.65 \%$ of the nurses). Only $17.7 \%$ of the physicians and $14.89 \%$ of the nurses are familiar with the principles of e-health. Only $21.8 \%$ of the physicians and $25.8 \%$ of the nurses consider that the country is ready for the implementation of e-health. Almost all, $90.4 \%$ of the physicians and $82 \%$ of the nurses recognize the need for EHRs. About half of the physicians (46.1\%) and $22.2 \%$ of the nurses would use an EHR system if they have adequate software. Physicians and nurses are not aware of what actually EHRs are. Most (74.5\%) of the physicians and 69.3\% of the nurses consider the implementation of personal health cards (presumably PHR), as a prerequisite for the implementation of e-health. The two preferred suggestions for the ownership of medical data are general practitioners (preferred by $28.3 \%$ of the physicians in $1999,30.9 \%$ - in 2013 , and $40.55 \%$ of the nurses) and the patient himself, preferred by $10.4 \%$ of the physicians in 1999, 29.38\% - in 2013 and $26 \%$ of the nurses.

Address for correspondence:

Assoc. prof. Iskra Mircheva

Department of Social Medicine and Healthcare Organization

Faculty of Public Health

Medical University of Varna

55 Marin Drinov St

9000 Varna, Bulgaria

e-mail:mircheva_is@abv.bg

Received: June 4, 2018

Accepted: June 14, 2018
CONCLUSIONS: Bulgarian physicians and nurses need additional education and training in using specialized, graphical and statistical software. The overall computer literacy of Bulgarian physicians and nurses is not adequate for the use of EHRs. They need additional education on the principles of eHealth, including EHRs, i.e. education in health informatics.

Keywords: physicians, nurses, e-health, EHRs, ownership of medical data 


\section{INTRODUCTION}

Healthcare is extremely important for the wellbeing of a country, both from an economic and social point of view, as well as from a demographic point of view. We live in a century when the health sector requires cost effectiveness and at the same time, improvement of the quality and access to health care (1). Information and communication technologies (ICT) applied to health and healthcare systems can increase their efficiency, improve the quality of life and unlock innovation in health markets (2).

It is well-known that telemedicine and e-health are changing the way healthcare is provided. These concepts are seen as a potential solution to overcome the loss of information (communications failures), avoiding re-evaluations and improving the quality of care and patient satisfaction (3). About $20 \%$ of physicians in Canada use e-health resources in their routine daily activities (4). The mobile health market in 2017 amounts to about $\$ 21$ billion, and is expected to reach $\$ 46$ billion in 2020 . The number of patients using telehealth applications is constantly increasing from 0.35 million people in 2013 , they are expected to reach 7 million in 2010. In 2013 the digital health market amounts to about $\$ 80$ billion, and is expected to reach $\$ 200$ billion in 2020 (5). In 2017, 57\% of all Internet activities of women and $49 \%$ of all Internet activities of men in the UK relate to checking for symptoms of medical conditions before consulting their physician ( $\left.{ }^{6}\right)$. Most of the Americans (91\%) and $88 \%$ of the Europeans own smartphones and $13 \%$ of them use healthcare applications for these phones (7). The number of downloaded mobile healthcare applications in 2017 is about 3.7 billion $\left(^{8}\right)$. It could be said that the health information received/sought via Internet could improve the patient-physician communication (9).

The WHO official definition formulated in Resolution WHA58.33 is: „eHealth is the cost-effective and secure use of information and communication technologies in support of health and health-related areas, including health services, health surveillance, health literacy and health education, health knowledge and research." (10).

E-health applications are numerous. They can be used for storing, managing and sharing data, i.e. health information exchange and interoperability, electronic health records, computer-assisted history taking systems (11), computerized physician order entry (CPOE) $(12,13)$, support of the everyday medical practice, and management of individual patients. eHealth applications can also be used to support clinical decision-making, i.e. computerized decision support systems (CDSS) (14-16), ePrescribing (17,18), epidemiological research and healthcare management activities (19). eHealth applications can also be used for delivering expert professional or consumer care remotely, i.e. telehealth and telemedicine (20). On the other hand, e-health cannot be implemented without the development and implementation of EHRs.

The relevance of EHRs and the importance of their application are reflected in the EU Vision of ehealth interoperability (21). The interoperability of e-health is directly related to the "interoperability of electronic health record (EHR) and the metrics to allow comparisons. EHRs can be a potentially valuable source of outcome data, if it is possible to harmonize the information in each EHR and accurately profile each patient to make valid and precise comparisons between fine-grained sub-populations."

According to the WHO (22), between 2010 and 2015, the deployment of national EHR systems has increased by $46 \%$ and already around half (47\%) of all WHO Member States have implemented a national EHR system. Most of these systems are integrated with laboratory, pharmacy and imaging systems.

The main barriers to the deployment of EHR systems are the lack of funding, infrastructure, capacity and legal framework, as well as the insufficient computer literacy of medical staff. The effective provision of healthcare as appropriate for the ehealth environment needs not only suitable buildings, modern medical equipment, and information and communication technologies, it also needs adequately trained healthcare staff able to use these technologies.

\section{AIM}

The aim of this article is to assess the computer literacy of Bulgarian physicians and nurses, their attitude to e-health, especially to EHRs as a major issue of e-health, including the ownership of medical data. 


\section{MATERIAL AND METHODS}

An enquiry comprising 33 questions (1 rank scaled with 5 levels of the scale and 32 questions with standardized answers) is used. The data is processed by means of IBM SPSS v.22 and STATISTICA. Apart from descriptive statistics, non-parametric tests are used to compare two or more nominal and ordinal scaled variables. Respondents of the study are physicians and nurses from five regional and university hospitals in the North-eastern region of Bulgaria (Varna, Shumen, Dobrich), who are employed in the hospitals during the period November - December, 2013.

Some of the results are compared to those of a study, carried out in 1999 among the physicians in Bulgaria, based on a similar questionnaire (23).

Several problems are discussed: self-evaluation of the experience in using computers, general attitude to eHealth, attitude to EHRs, including ownership of medical data. Other features, like legal issues, confidentiality, etc. had already been partially presented in other publications $(23,24)$. The evaluation of using computers is based on self-evaluation of the experience in using Internet, basic computer skills, medical information systems and data bases (MIS and DB), specialized, graphical and statistical software.

\section{RESULTS AND DISCUSSION}

\section{Characteristics of the Samples}

The number of the participating physicians is 271. The response rate is $82.15 \%$ (330 enquiries were sent, 271 were filled out and returned). The study covers $10.98 \%$ of all physicians in the region. The distribution of the respondents by gender is as follows: $46.9 \%$ males and $53.1 \%$ females. The largest group is that of physicians aged 50-54 years (17.71\%), followed by the age group of $40-44$ years (15.5\%) and the smallest is 60 years and above $(4.43 \%)$. The young specialists (younger than 30 years) are $9.96 \%$. The other age groups are almost equal (between 13 and $14 \%)$. About half of the respondents are employed in the two university hospitals in Varna $(29.15 \%$ in St. Marina University Hospital and $26.57 \%$ in St. Anna University Hospital). The structure of the sample correlates with the structure of all clinicians by specialization in Bulgaria (Wilcoxon Signed Ranks Test: $\mathrm{z}=-2.934$, Asymp. Sig. (2-tailed $)=0.003)(\mathrm{r}=0.999$, $\mathrm{p}=0.031$ ).

The number of the participating nurses is 423 . The response rate is (81.35\%) (520 enquiries were sent, 423 were filled out and returned). The study covers $10.78 \%$ nurses in the region. All participants are female. The largest group is that of nurses aged 40-44 years (20.8\%) and the professionals below 30 years of age are $15.37 \%$. The smallest group is 60 years of age and above (2.36\%). About half of the respondents (43.7\%) are employed in the two university hospitals in Varna. The structure of the sample correlates with the structure of all nurses by specialization in Bulgaria (Wilcoxon Signed Ranks Test: $\mathrm{z}=$ 2.023, Asymp. Sig. (2-tailed) = 0.043) .

Cronbach's alpha as a measure of internal consistency of the questionnaires used in both studies is 0.798 for the study from 1999 and 0.832 for the study from 2013.

Self-Evaluation of the Experience in Using

\section{Computers}

The overall self-evaluation in using computers is 4.16 for physicians and 3.74 for nurses ( $\max =6.00$ ). As expected, it is highest in using Internet (physicians - 4.83, nurses - 4.4), followed by basic computer skills (physicians - 4.71, nurses - 4.23), management information systems (MISs) and databases (DBs) (physicians - 4.11, nurses - 3.72), specialized software (physicians - 4.08, nurses - 3.59), and lowest in using statistical software (physicians - 3.57, nurses - 3.28) and graphical software (physicians - 3,64, nurses 3,19) (Fig. 1). A statistically significant difference is found between the evaluation of the differ-

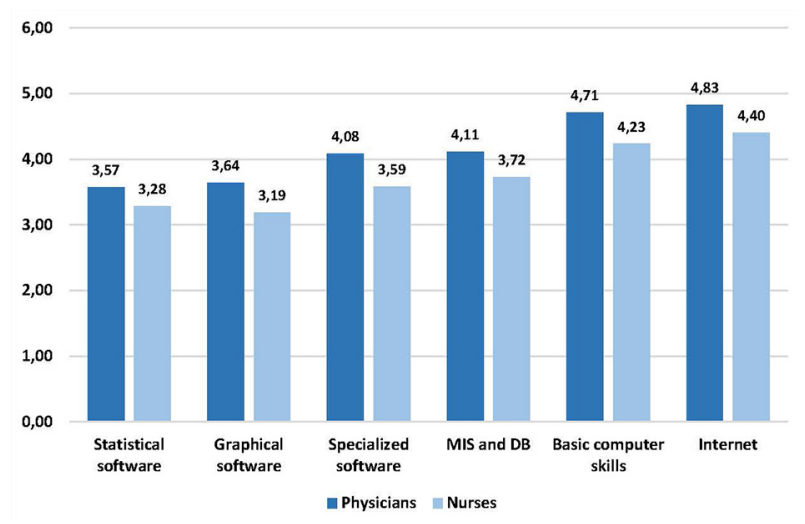

Fig. 1. Self-evaluation of the experience in using computers by Bulgarian physicians and nurses (max $=6.0$ ) 
ent computer skills both for the physicians $(\mathrm{F}=4.917$, $\mathrm{p}=0.005)$ and for the nurses $(\mathrm{F}=4.535, \mathrm{p}=0.007)$.

Although the overall evaluation is between sufficient and very good, never reaching very good (5.00), it is considered as a sufficient ability to use computers by $74.5 \%$ of the physicians and $63.3 \%$ of the nurses (in our 1999 study only $19.6 \%$ of the physicians declared their sufficient abilities to use computers) (23).

Obviously, what physicians best know is how to use the Internet, as only a small fraction of them (11.81\%) have indicated that they need additional training to use the Internet. Around a quarter (26.57\%) need additional training in using word processing and spreadsheets, $37.64 \%$ need additional training in using data bases and information systems (medical, clinical), 40.59\% need additional training in using graphical software. The greatest need is for additional training in the use of statistical (47.98\%) and specialized software (48.34\%). Similarly, only $14.18 \%$ of the nurses need additional training to use the Internet. The relative share of those who need additional training in the other skills under consideration is much higher: training in word processing and spreadsheets is needed by $35.93 \%$ of the nurses, DB and MIS - 38.3\%, graphical software - $42.32 \%$. The need for training in using statistical software is declared by $39.24 \%$. The need for specialized software training (49.65\%) is the highest (Fig. 2).

It is evident that training in specialized software can hardly be achieved within the education of future doctors and nurses. This is not the case with the training to use statistical and graphical software. Medical statistics is included in the curriculum of medical students. The number of hours for this discipline is extremely inadequate, and the fact that it is taught in the second semester of the medical education diminishes its meaning. This is obviously a flaw in the medical education. Informatics is offered to medical students as an elective discipline, but is rarely chosen. Informatics as a regular discipline is included in the nursing curriculum and covers basic computer literacy - word processing, spreadsheets, preparation of presentations. Medical statistics is offered as an elective discipline to nurses, never chosen by the students.

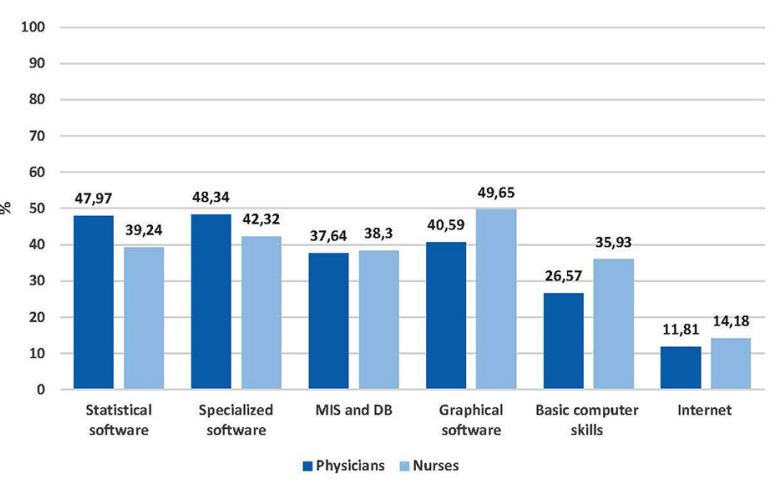

Fig. 2. Need for additional education and training in using computers

\section{Attitude to $e$-Health}

The term e-health has been in use since 1999 as a modern slang describing the use of computers and the Internet in medicine. Although almost constantly speaking and writing about e-health and its implementation, medical professionals are not aware of its principles. The proportion of physicians in our study who are familiar with the principles of e-health is relatively low - only $17.7 \%$ of the respondents. Even lower is the proportion of nurses who are familiar with the principles of e-health (14.89\%). This corresponds to the low rates of those who consider that the country is ready for the implementation of e-health $(21.8 \%$ of the physicians and $25.8 \%$ of the nurses). No statistically significant difference in the opinions of physicians and nurses is found $\left(\mathrm{X}^{2}=-.975, \mathrm{p}=0.323\right.$ for the principles, and $1.436, \mathrm{p}=0.231$ for the readiness). Considering that our study is conducted in large city hospitals, we can assume that these proportions will be even lower if we standardize the answers received to all physicians and nurses in the country. There is no statistically significant relation between knowing the principles of e-health and profession, gender, age and place of work of the respondents.

\section{Attitude to EHRs}

Almost all, $90.4 \%$ of the physicians and $82 \%$ of the nurses recognize the need for electronic health records. However, about half of the physicians (52.4\%) and $58.9 \%$ of the nurses state that they do not have an electronic health record system in their clinic. This is a matter of health policy, which needs to be solved on a national level.

About half of the physicians (46.1\%) and only $22.2 \%$ of the nurses would use an electronic health 
record system if they have adequate software, yet, about one third of the physicians (28.4\%) and 60.5\% of the nurses would use such a system only if they are forced to do that. Only $25.5 \%$ of the physicians and $17.3 \%$ of the nurses would use such a system if they are provided with adequate software for free. In our study in 1999, only $9.5 \%$ of the physicians would use EHRs if they were forced to do that (Fig. 3).

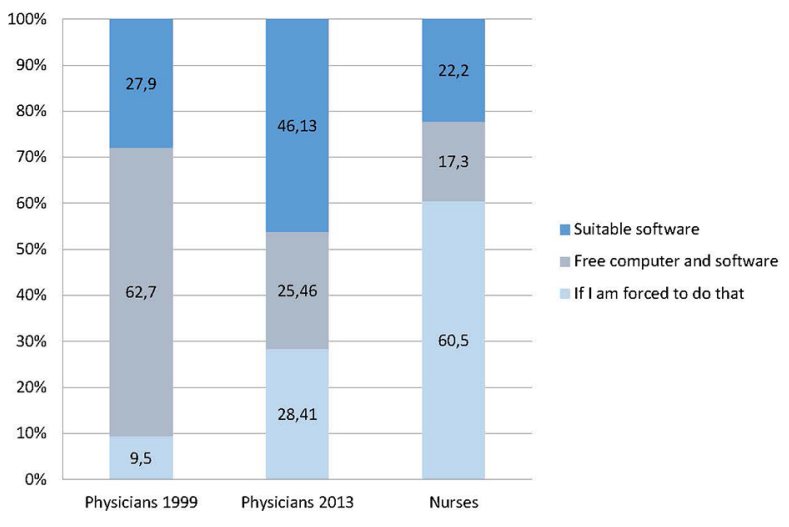

Fig. 3. Conditions implied to the use of EHRs

Neither physicians, nor nurses are willing to enter patient data into a computer. This is done by only $38.38 \%$ of the physicians $(22.51 \%$ believing that they would have a complete view of the patient's health status and $15.87 \%$ believing that they would save time). It is alarming that $35.06 \%$ consider that this is not their job. Similar are the responses of the nurses, where only $28.8 \%$ of the nurses are willing to enter their patients'data into a computer (13\% consider that they will have a complete view of the patient's health status and $15.8 \%$ think that this will save them time). A little less than half of the nurses (43.3\%) think it is not their job. Probably both physicians and nurses do not want or are insufficiently prepared to deal with such „unusual“ activities themselves.

In 1999, the answers to a similar question are quite different: $59.1 \%$ of the physicians are willing to enter patient's data by themselves as this will provide a comprehensive view of the health status of their patients, or will save them time (17.5\%) and $18.7 \%$ state that this is not their job. If we summarize and compare the responses (Fig. 4), in 1999, 76.6\% of the interviewed physicians have declared willingness to personally enter medical data into a computer, and only $38.38 \%$ do that in 2012 . The share of the nurses

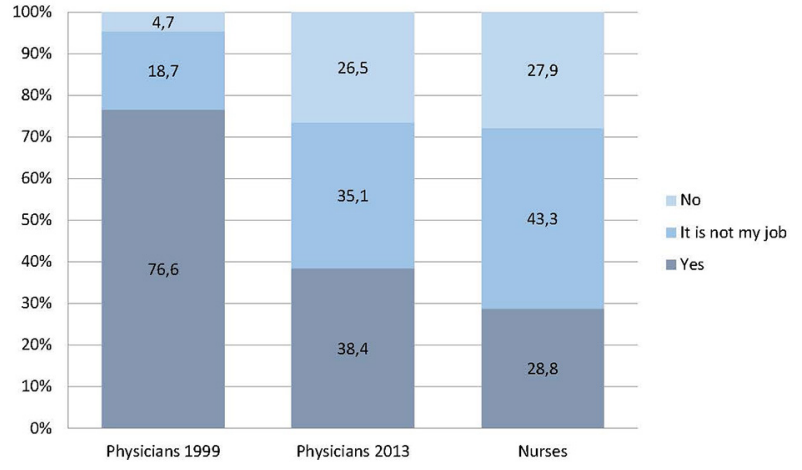

Fig. 4. Willingness to personally enter medical data into a computer

is still lower (28.8\%).

Our opinion is that the answers to this question in 2013 as well as in 1999, confirm our hypothesis that the issue on the development and the implementation of certified medical software, including EHR software is still pending and should be considered by the corresponding health and financial authorities. Another issue is the motivation of medical professionals to use computers and specialized software in their daily practice, and not just surfing the Internet.

The opinion of the physicians and the nurses differs significantly concerning EHRs. Physicians are more likely to accept the necessity of EHRs $\left(X^{2}=9.223, p=0.002\right)$; the use of common criteria on the structure and the contents of the electronic health records $\left(\mathrm{X}^{2}=15.062, \mathrm{p}=0.000\right)$ and the inclusion of nursing data in EHRs $\left(\mathrm{X}^{2}=3.999, \mathrm{p}=0.045\right)$.

Neither physicians, nor nurses are aware of what actually an electronic health record is. The majority $(74.5 \%)$ of the physicians and $69.3 \%$ of the nurses consider the implementation of personal health cards (presumably personal health records), as a prerequisite for the implementation of e-health and the improvement of the quality of healthcare and healthcare organization. There is no consensus on the contents of the personal health record. The majority of the respondents $(91.5 \%$ of physicians and $87 \%$ of nurses) consider that it should include the complete EHR; health insurance data is the choice of $69 \%$ of the physicians and $52.2 \%$ of the nurses; demographic data (59.4\% of physicians and $43 \%$ of nurses); last episode of illness $(50.2 \%$ of physicians and $32 \%$ of nurses) (Fig. 5). Again, the contradictory answers give the idea that Bulgarian physicians and nurses are not acquainted neither with e-health nor with electronic 
health records. The differences in the attitude of the physicians and the nurses on the content of the PHRs are statistically significant $(\mathrm{p}=0.000)$, except for the complete EHRs ( $\mathrm{p}=0.066)$.

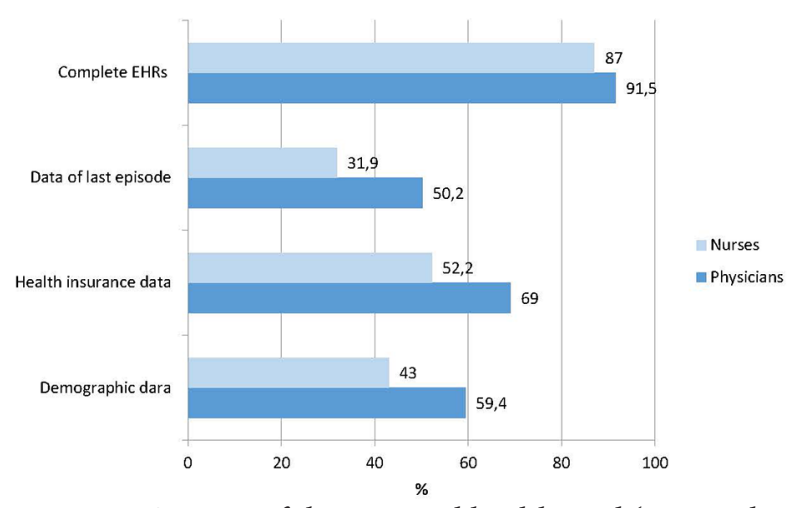

Fig. 5. Content of the Personal health card (personal health record)

\section{Ownership of Medical Data}

Considering the importance of the issue on ownership of medical data, a question is intentionally used in both studies. The question is very simply asked without considering any moral, ethical or legal aspects of the problem. Our aim is to just ask about the opinion of the respondents as a general concern on the ownership of medical data.

Perhaps, that is why $25 \%$ of the participants do not respond to this question in 1999, while the other answers have 44 different meanings, of which only $9(\mathrm{p}<0.005)$ are significant. In 2013, all participants respond by indicating more than one response. As a result, their answers have 22 different meanings, of which 15 are statistically significant $(\mathrm{p}<0.05)$. Part of the answers are presented in Fig. 6.

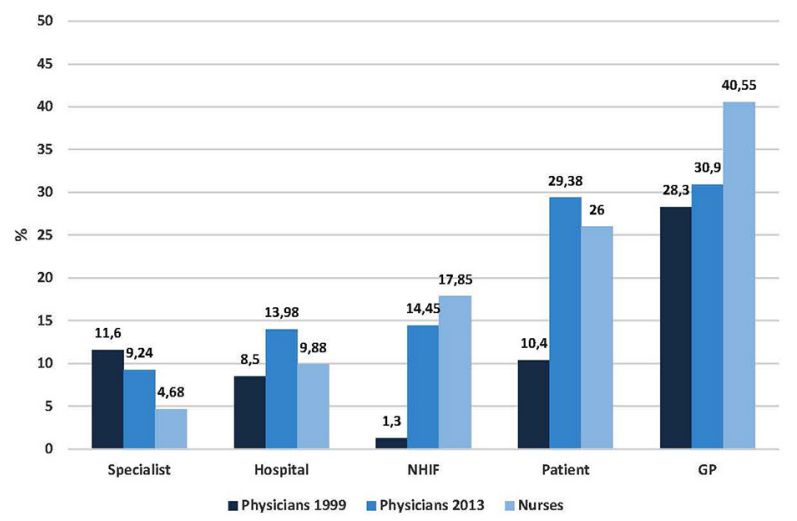

Fig. 6. Concerns on ownership of medical data
In $1999,28.3 \%$ of the physicians consider that the owner of the patient's medical data should be their general practitioner. In 2013, this proportion is higher (30.9\%). For nurses, it is even higher - $40.55 \%$. Assuming that it is so, it would not be so important to focus on the privacy of the patient's data and the right to decide for themselves to whom to reveal this data. On the other hand, account should also be taken of the fact that the EHR is distributed by architecture, i.e. parts of it may be stored on different computer systems, i.e. in the clinics where the relevant medical data emerges. Consequently, although the GP has access to this data, it is virtually illogical, superfluous, and even impossible for the GP to keep the entire EHRs of her/his patients. Therefore, the idea that the patient's medical data is owned by the general practitioner is not feasible.

The second proposal, made by $29.38 \%$ of the physicians in 2013 and $10.4 \%$ of the physicians in the 1999 surveys, is that the owner of the patient's medical data should be the patient herself/himself. The share of nurses who support this idea (26\%) is also relatively high. Our opinion is that this option is more logical than the previous and seems the most plausible. The source of medical data is the patient and the data is related to his/her personality and his/ her health status, i.e. it is quite logical to assume that this is personal data of the patient and the patient should own it. Indeed, the data is obtained with the active participation of medical professionals - physicians, nurses, laboratory technicians, etc. It could be said that this data could not have existed without the involvement of medical professionals, but this, on one hand, is an obligation of the medical specialists, and on the other hand, the medical professionals are paid for the delivery of these "health services" or "health care". Therefore, the patient's medical data is his/her personal data and as such is his/her property. Another issue is the ownership of the physical medium on which this data is stored. In any case, it remains a property of the health care facility or the health care professional.

The proportion of physicians suggesting the hospital as the owner of the medical data is relatively small: $13.98 \%$ in 2013 and $8.5 \%$ in 1999. Similarly, this is the choice of $9.88 \%$ of the nurses. This is not impossible with modern technologies, but it seems 
unreal, as it involves treating the patient only in a well-defined hospital. This limits the patient's right to choose a medical establishment. We assume that these answers are dictated by the fact that the surveys were conducted in hospitals.

There is a small proportion of those who consider that the owner of the patient's medical data should be a specialist from the outpatient care (11.6\% in $1999,9.24 \%$ in 2013 and $4.68 \%$ of nurses). Our opinion is that this option, apart from being illogical, cannot practically be achieved, it could concern patients who are chronically ill and who rely on the health care provided by a particular specialist, but this is only a special case and does not concern all patients.

Except for the National Health Insurance Fund (NHIF) data, all differences in the answers between physicians and nurses are statistically significant $(\mathrm{p}<0.03)$.

In 1999 , a proposal is made by $8.5 \%$ of the respondents, according to which the owner of the medical data of the patient should be a health institution without specifying the type of the health establishment. There is no such response in the 2013 survey. Rather, we can speak of a collective (aggregate) image of all the healthcare facilities the patient has been visiting, starting with the GP, a specialist, a hospital, and so on. This is interesting as an idea, but considering the varied ownership of healthcare facilities, we can hardly identify the owner of the patient's medical data.

Combined responses as "general practitioner plus patient" or "physician plus patient" can be assigned to any of the above options. The options "general practitioner plus physician“ and „general practitioner plus a health care facility" are meaningless as they exclude the patient involvement.

We would also identify the option "health insurance fund" as pointless as it does not include the patient's involvement. This option was proposed by $1.3 \%$ of the physicians in $1999,14.45 \%$ of the physicians in 2013 and $17.85 \%$ of the nurses. Obviously, the Health Insurance Fund, as well as other health organizations, may use some of the patient's medical data, even without his or her consent, but this is data needed to shape the financial and accounting part of health care, and in no case these organizations are entitled to some ownership of the patient's medical data.

In summary, due to insufficient knowledge of EHRs, the principles of e-health and relatively poor computer literacy, Bulgarian physicians and nurses cannot identify the owner of medical data.

\section{Practical Considerations}

In 2010, the Bulgarian NHIF's personalized information system (PIS) comprising personal electronic health records, has been introduced. The system reflects all NHIF-paid services - medical exams, drugs prescriptions, hospitalizations of each health insured person within the healthcare system. Access to it is through the site of the National Health Insurance Fund, initially only for those that have an electronic signature, which at that time made the system not quite usable. From the beginning of 2013, the NHIF's personalized information system can be used through a unique access code issued by the regional health insurance funds. The right of access to the NHIF's personalized information system through this code is granted to all health insured individuals within the meaning of the Health Insurance Act, including the individuals whose health insurance rights are interrupted.

It should be noted that the information stored and maintained by the NHIF cannot be called the patient's „electronic medical record“ as it does not correspond to the content of any of the major types of electronic medical records originally accepted and used in the United States, subsequently in $\mathrm{Eu}-$ rope and other countries around the world. In 2005, the International Standards Organization in its ISO / TR 20514 standard (25) sets out the definitions of the three types of medical records that were later elaborated in the United States by the National Allianace of Health Information Technology (NAHIT) (26), namely:

$\diamond$ An Electronic medical record: An electronic record of health-related information on an individual that can be created, gathered, managed and consulted by authorized clinicians and staff within one health care organization.

$\diamond$ An Electronic health record: An electronic record of health-related information on an individual that conforms to nationally accepted interoperability standards and that can be creat- 
ed, managed and consulted by authorized clinicians and staff in more than one health care organization.

$\diamond$ A Personal health record: an electronic record of health-related information on an individual that conforms to nationally accepted interoperability standards and that can be drawn from multiple sources while being managed, shared and controlled by the individual.

The European Union uses somewhat different terminology, which according to the official documents is as follows:

$\diamond$ "electronic health record" means a comprehensive medical record or similar documentation of the past and present physical and mental state of health of an individual in electronic form, and providing for ready availability of these data for medical treatment and other closely related purposes;

Considering the above-cited definitions, it is difficult to decide what is actually the record, maintained by NHIF. It covers mostly administrative and financial data, a little bit of diagnosis, prescriptions and hospitalizations. Perhaps it should be called something like "administrative" or "financial" patient record. On the other hand, since this record is accessible by the patient it is supposed to be more or less a personal health record. If this is a personal health record, the patient should have the right to manipulate it, which in this case is quite impossible. Another issue is that NHIF cannot maintain the entire electronic health records of all the patients. However, this is an issue that is not a subject of this publication.

\section{CONCLUSION}

$\diamond$ The computer literacy of Bulgarian physicians and nurses is adequate in using Internet and basic computer skills (word-processing and electronic tables). However, they need additional education and training in using specialized, graphical and statistical software.

$\diamond$ The overall computer literacy of Bulgarian physicians and nurses is not adequate for using EHRs.

$\diamond$ Bulgarian physicians and nurses need additional education on the principles of eHealth, including such important e-health issues as EHRs, which means that they need education in health informatics.

$\diamond$ The knowledge in the area of health informatics and e-health should contribute to the development and implementation of real EHRs used appropriately by medical professionals and patients.

\section{REFERENCES}

1. Docteur E, Oxley H. Health care systems: the lessons from the reform experience, OECD working papers. OECD: Paris; 2003.

2. Communication from the Commission to the $\mathrm{Eu}-$ ropean Parliament, the Council, the European Economic and Social Committee and the Committee of the Regions eHealth Action Plan 2012-2020 - Innovative healthcare for the 21st century, Brussels, 6.12.2012, $\operatorname{COM}(2012) 736$ final.

3. Kruse CS, Krowski N, Rodriguez B, Tran L, Vela J, Brooks M. Telehealth and patient satisfaction: a systematic review and narrative analysis. BMJ Open. 2017; 7 (8):e016242. doi: 10.1136/ bmjopen-2017-016242.

4. https://www.cma.ca/En/Pages/The-digital-worldGood-or-bad-for-MDs.aspx. accessed 2.05.2018

5. https://www.statista.com/statistics/, accessed 25.05.2018

6. https://www.statista.com/statistics/286079/internet-activities-in-great-britain-by-gender/ accessed: 16.05.2018

7. Fox S, Duggan M. Health Online 2013. Washington, D.C.: Pew Research Center. 2013.

8. https://www.statista.com/statistics/625034/mobilehealth-app-downloads/ accessed 16.05.2018

9. Tan SSL, Goonawardene N. Internet health information seeking and the patient-physician relationship: A systematic review. J Med Internet Res. 2017; 19(1): e9. doi:10.2196/jmir.5729.

10. Resolution WHA58.33. Sustainable health financing, universal coverage and social health insurance. In: Fifty-eighth World Health Assembly, Geneva, 16-25 May 2005. Resolutions and decisions annex. Geneva: World Health Organization; 2005.

11. Pringle M. Using computers to take patient histories. BMJ. 1988;297(6650):697-8.

12. Holdsworth MT, Fichtl RE, Raisch DW, Hewryk A, Behta M, Mendez-Rico E, et al. Impact of comput- 
erized prescriber order entry on the incidence of adverse drug events in pediatric inpatients. Pediatrics. 2007;120(5):1058-66.

13. Bates DW. Computerized physician order entry and medication errors: finding a balance. J Biomed Inform. 2005; 38(4):259-61. doi: 10.1016/j. jbi.2005.05.003.

14. Bates DW, Kuperman GJ, Wang S, Gandhi T, Kittler A, Volk L, et al. Ten commandments for effective clinical decision support: making the practice of evidence-based medicine a reality. J Am Med Inform Assoc. 2003;10(6):523-30. doi: 10.1197/jamia. M1370.

15. Garg AX, Adhikari NKJ, McDonald H, RosasArellano MP, Devereaux PJ, Beyene J et al. Effects of computerized clinical decision support systems on practitioner performance and patient outcomes: a systematic review. JAMA. 2005;293(10):1223-38. doi: 10.1001/jama.293.10.1223.

16. Kuperman GJ, Bobb AM, Payne T, Avery AJ, Gandhi TK, Burns G, et al. Medication-related clinical decision support in computerized provider order entry systems: a review. J Am Med Inform Assoc. 2007;14(1):29-40. doi:10.1197/jamia.M2170.

17. Barber N, Cornford T, Klecun E. Qualitative evaluation of an electronic prescribing and administration system. Qual Saf Health Care. 2007;16(4):2718. doi: 10.1136/qshc.2006.019505.

18. Bates DW. Preventing medication errors: a summary. Am J Health Syst Pharm. 2007;64(14 Suppl 9):S3-9. doi: 10.2146/ajhp070190.

19. Pagliari C, Sloan D, Gregor P, Sullivan F, Kahan JP, Detmer D, et al. Literature review and conceptual map of the field of eHealth. Final report to the SDO programme; 2004.

20. Hersh WR, Hickam DH, Severance SM, Dana TL, Pyle KK, Helfand M. Diagnosis, access and outcomes: Update of a systematic review of telemedicine services. J Telemed Telecare. 2006;12(S2):S3-31. doi: 10.1258/135763306778393117.

21. van Langenhove $P$, Decreus K, Rogala A, Olyslaegers T, Whitehouse D. eHealth European Interoperability Framework. Vision on eHealth EIF, a study prepared for the European Commission by the Deloitte team, Version 1.2, EC, Brussels; 2013.

22. WHO. Global diffusion of eHealth: making universal health coverage achievable. Report of the third global survey on eHealth. Geneva; 2016.
23. Feschieva N, Mircheva I. Proofs of the necessity of medical informatics for the physicians in Bulgaria, In: Patel V, Rogers R, Haux R, editors. Proceedings of MedInfo 2001, Vol.84 Studies in Health Technology and Informatics, IOS Press, The Netherlands, part two; 1019:1023; 2001.

24. Mircheva I. E-health and the need for education in biomedical and health informatics for the physicians in Bulgaria. Scr Sci Salutis Publicae. 2016; 2(1):23-9.

25. Technical report Health informatics - Electronic health record - Definition, scope and context, ISO/TR 20514; 2005.

26. The National Alliance for health information technology. Report to the Office of the national coordinator for health information technology on Defining key health information technology terms. April $28,2008$. 